\title{
ATRIBUTOS BIOFÍSICOS DE FRUTOS E SEMENTES E EMERGÊNCIA DE PLÂNTULAS DE JUTAÍ-AÇÚ
}

Marcus Victor Almeida Campos ${ }^{1}$; Noemi Vianna Martins Leão ${ }^{2}$; Elizabeth Santos Cordeiro Shimizu ${ }^{3}$; Alessandra Doce Dias de Freitas ${ }^{4}$; Sérgio Heitor Sousa Felipe ${ }^{5}$

${ }^{1}$ Graduado em Engenharia Ambiental, Universidade do Estado do Pará, Belém, Pará, Brasil.marcusvictor.campos@gmail.com.

${ }^{2}$ Pesquisadora do Laboratório de Sementes Florestais, Embrapa Amazônia Oriental, Belém, Pará, Brasil.

${ }^{3}$ Analista do Laboratório de Sementes Florestais, Embrapa Amazônia Oriental, Belém, Pará, Brasil.

${ }^{4}$ Docente da Faculdade de Engenharia Florestal, Universidade Federal do Pará, Altamira, Pará, Brasil.

${ }^{5}$ Doutorando do curso de Botânica da Universidade Federal de Viçosa, Viçosa, Minas Gerais, Brasil.

Recebido em: 06/04/2018 - Aprovado em: 10/06/2018 - Publicado em: 20/06/2018 DOI: 10.18677/EnciBio_2018A12

Estudos biométricos de frutos e sementes podem detectar variabilidade fenotípica entre indivíduos da mesma espécie e serem aplicados em programas de melhoramento genético. Além disso, contribuem para a seleção de sementes com maior padrão de qualidade biofísica a serem destinadas à produção de mudas. $\mathrm{O}$ trabalho objetivou avaliar os atributos biofísicos de frutos e sementes e emergência de plântulas de diferentes procedências de jutaí-açú (Hymenaea courbaril L.). Os frutos e sementes são procedentes dos municípios de Ananindeua e Portel, Pará. Para a avaliação biométrica de frutos e sementes e massa fresca foram utilizados 50 e 100 unidades, respectivamente. Adicionalmente, avaliou-se o grau de umidade e número de sementes por fruto. $O$ teste de emergência foi realizado em delineamento inteiramente ao acaso, constando de quatro repetições de 25 sementes. Avaliou-se a porcentagem de emergência (E), índice de velocidade de emergência (IVE) e tempo médio de emergência (TME). A procedência de Ananindeua apresentou maiores médias para comprimento, espessura e número de sementes por fruto. Em contraste, a procedência de Portel apresentou as maiores médias para comprimento e espessura das sementes. A procedência de Ananindeua foi superior fisiologicamente em relação à procedência Portel, sendo E igual a 73 e $21 \%$, respectivamente, o TME foi 14,86 e 23,03, respectivamente, e o IVE foi 1,27 e 0,23 , respectivamente. Os resultados evidenciam variações entre os atributos biofísicos e fisiológicos das duas procedências.

PALAVRAS-CHAVE: diversidade morfométrica, Hymenaea courbaril, massa fresca 


\title{
FRUIT AND SEEDS BIOPHYSICAL TRAITS AND EMERGENCY OF "JUTAÍ-AÇÚ" SEEDLINGS
}

\begin{abstract}
Biometric studies of fruits and seeds can detect phenotypic variability among individuals of the same species and it be applied in breeding programs. Besides, they contribute to the selection of seeds with a higher standard of biophysical quality to be used for the seedlings production. The objective of this study was to evaluate the fruit and seeds biophysical traits and emergency of "jutaí-açú" (Hymenaea courbaril L.) seedlings from different provenance. The fruits and seeds come from the municipalities of Ananindeua and Portel, Pará. For biometric evaluation of fruits and seeds and fresh mass, 50 and 100 units were used, respectively. Additionally, the moisture content and number of seeds per fruit were evaluated. The emergency test was performed in a completely randomized design, consisting four replicates of 25 seeds each. The percentage of emergency (E), emergency speed index (ESI) and average time of emergency (ATE) were evaluated. Ananindeua provenance showed higher averages for length, thickness and number of seeds per fruit. In contrast, the Portel provenance showed the highest averages for length and seed thickness. Ananindeua provenance was higher physiologically in relation to the Portel provenance, with $E$ equal to 73 and 21\%, respectively; the TME was 14.86 and 23.03, respectively; and the IVE was 1.27 and 0.23 respectively. The results showed variations between the biophysical and physiological attributes of the two provenances.
\end{abstract}

KEYWORDS: morphometric diversity, Hymenaea courbaril, fresh mass

\section{INTRODUÇÃO}

Jutaí-açú ou jatobá (Hymenaea courbaril L.) pertencente à família Fabaceae é uma espécie estratégica para uso na restauração florestal, além de apresentar frutos com grande aceitação para a fauna (MARTINS, 2013). Sua madeira é considerada resistente, tendo como principais utilizações a construção civil, postes, estacas, móveis e laminados (CIPRIANO et al., 2014).

A espécie ocorre nos biomas brasileiros como Amazônia, Caatinga, Cerrado, Mata Atlântica, Pantanal, com exceção dos Pampas (LUCENA et al., 2016), em floresta semidecídua (GONZAGA et al., 2016), atingindo de 30 a $40 \mathrm{~m}$ de altura, em solos de média a alta fertilidade, podendo ocorrer em várzeas altas, com frequência em solos argilosos ou pobres (SILVA; LEÃO, 2006). Os frutos são muito procurados pela fauna e pelo homem, devido as sementes serem envolvidas por uma farinha comestível e nutritiva (LORENZI, 2002). Estudos sobre a biometria de frutos e sementes inferem sobre a variabilidade genética entre indivíduos da mesma espécie, e a correlação entre esta variabilidade e os fatores ambientais (GONÇALVES et al., 2013).

Pesquisas relacionadas a influência da dimensão das sementes e frutos na qualidade fisiológica tem sido bastante investigada para as espécies agrícolas, contudo, ainda existe pouca pesquisa para as essências florestais nativas do Brasil (DUTRA, et al., 2017). Segundo Freitas et al. (2014) esses estudos ainda contribuem para elaboração de novas técnicas sustentáveis e para subsidiar a maximização dos recursos por meio da propagação de sementes de espécies florestais.

A emergência de plântulas torna-se um processo de conhecimento importante para a preservação das comunidades vegetais, assim como para as pesquisas relacionadas à conservação genética e propagação das espécies (SMIDERLE; 
SOUZA, 2003; KOSZO, 2006). Desse modo, considerando a importância das espécies em programas de recuperação de áreas degradadas, o objetivo do trabalho foi avaliar os atributos biofísicos de frutos e sementes e emergência de plântulas de jutaí-açú.

\section{MATERIAL E MÉTODOS}

O estudo foi desenvolvido no Laboratório de Sementes Florestais (LASF) da Embrapa Amazônia Oriental, Belém, PA, coordenadas de 01²6'12.8" latitude S e 48 26'33.8" longitude W a $35 \mathrm{~m}$ de altitude. A classificação climática, segundo Köppen, é do tipo AF, com temperatura média anual de $26^{\circ} \mathrm{C}$ (FAPESPA, 2016).

Os frutos foram coletados em agosto e setembro de 2016, em duas procedências localizadas, respectivamente nos municípios de Portel-PA $\left(2^{\circ} 04^{\prime} 30.6 " S 50^{\circ} 41^{\prime} 07.5^{\prime \prime} \mathrm{W}\right)$ e Ananindeua-PA (1 $\left.{ }^{\circ} 21^{\prime} 08.0^{\prime \prime S} 48^{\circ} 25^{\prime} 01.1^{\prime \prime W}\right)$, com altitudes equivalentes a 19 e 20 m, respectivamente. A classificação Köppen indica um clima tropical úmido tipo AF, megatérmico para os dois municípios, sendo Portel com temperatura média anual de $26^{\circ} \mathrm{C}$, e Ananindeua com média de $25^{\circ} \mathrm{C}$ (FAPESPA, 2016).

Foram selecionados aleatoriamente 50 frutos, por procedência, para medição do comprimento, largura e espessura. Além disso, avaliou-se o número de sementes por fruto e a massa fresca. Para a biometria das sementes, foram utilizadas 100 unidades por procedência, considerando os mesmos parâmetros. Para as medições de massa fresca utilizou-se balança analítica com precisão de 0,001 g, e, para as medições biométricas utilizou-se paquímetro digital, com $0,01 \mathrm{~mm}$ de precisão.

Foram determinados o número de sementes por quilograma $(\mathrm{kg})$ e o grau de umidade das sementes pelo método de estufa à $105 \pm 3^{\circ} \mathrm{C}$ (BRASIL, 2009). Para a determinação do grau de umidade as sementes foram cortadas ao meio no sentido longitudinal ao hilo.

As sementes foram submetidas ao tratamento pré-germinativo para a quebra da dormência tegumentar através da escarificação das laterais da semente, na região oposta ao hilo, utilizando-se um esmeril elétrico. Posteriormente, as sementes foram distribuídas em bandejas, contendo areia e serragem esterilizadas na proporção 1:1. O teste de emergência foi realizado em delineamento inteiramente ao acaso, constando de quatro repetições de 25 sementes. O experimento foi mantido em local semi-sombreado e irrigado duas vezes ao dia, sendo realizada a contagem diária do número de plântulas. Os parâmetros de emergência avaliados foram a porcentagem de emergência $(E)$, tempo médio de emergência (TME) e o índice de velocidade de emergência (IVE), representados pelas equações 1,2 e 3 , respectivamente:

$$
E=\left(\sum_{E} / \sum s\right)^{*} 100
$$

em que:

$E=$ Porcentagem de Emergência. 
$\sum_{\mathrm{E}}$ o o somatório de sementes emergidas até o momento da estabilização.

$\sum s=0$ somatório de sementes colocadas para emergir.

TME $=\left(N_{1} T_{1}+N_{2} T_{2}+\ldots+N_{n} T_{n}\right) /\left(N_{1}+N_{2}+\ldots+N_{n}\right)$

em que:

TME = Tempo Médio de emergência.

$N_{1}, N_{2}, N_{n}=0$ número de sementes emergidas nos tempos $T_{1}, T_{2}$ e $T_{n}$, respectivamente.

IVE $=\left(E_{1} / N_{1}\right)+\left(E_{2}+N_{2}\right)+\ldots+\left(E_{n}+N_{n}\right)$

em que:

IVE = Índice de Velocidade de Emergência.

$E_{1}, E_{2}$ e em = o número de sementes emergidas na primeira, segunda e última contagem.

$\mathrm{N}_{1}, \mathrm{~N}_{2}, \mathrm{~N}_{\mathrm{n}}$ = o número de dias decorridos da semeadura na primeira, segunda e última contagem.

Os dados foram submetidos à análise de variância (ANOVA), sendo as médias comparadas pelo teste de Tukey, ao nível de $5 \%(p<0,05)$. As análises estatísticas foram realizadas através do software ASSISTAT 7.7 ®.

\section{RESULTADOS E DISCUSSÃO}

Os valores médios para o teor de umidade de sementes das procedências de Portel e Ananindeua foram iguais a $7,77 \%$ e $11,42 \%$, respectivamente. Segundo Lima et al. (2017), o grau de umidade das sementes pode influenciar negativamente na germinação das mesmas, uma vez que baixos teores, em determinados casos, podem causar a morte do embrião. Além disso, é uma variável imprescindível em testes de laboratórios podendo influenciar diretamente no número de sementes por quilograma (SARMENTO et al., 2015).

O número de sementes por quilograma foi igual a 318 e 295 unidades para Ananindeua e Portel, respectivamente. Essa informação pode ser utilizada para planejar e estimar o potencial número de mudas, após o conhecimento da porcentagem de germinação. Adicionalmente, esse conhecimento é aplicado diretamente no comércio de sementes (BRASIL, 2009).

Os frutos evidenciaram diferença significativa entre as procedências em relação ao comprimento (Figura $1 \mathrm{~A}$ ), espessura (Figura 1B), número de semente por fruto (Figura 1E), sendo nestes casos a procedência de Ananindeua com as maiores médias iguais a 112,12 mm, 38,38 mm e 6,82 unidades, respectivamente. Esses resultados são indicativos de variações fenotípicas entre as procedências avaliadas que podem estar relacionadas à fatores edafoclimáticos e genéticos. Contudo, em 
relação à largura (Figura 1C), massa fresca (Figura 1D) e massa fresca de sementes por fruto (Figura 1F), não houve diferença significativa entre as procedências.

Estudos realizados por Zuffo et al. (2014) com frutos e sementes de Dipteryx alata Vog, evidenciaram a variabilidade fenotípica entre as procedências estudadas, corroborando com o presente estudo. Segundo Pereira et al. (2015) e Duarte et al. (2012), a procedência é um fator que influencia nas características biométricas de frutos e sementes, pois estas apresentam variabilidade em função das condições ambientais durante sua formação.
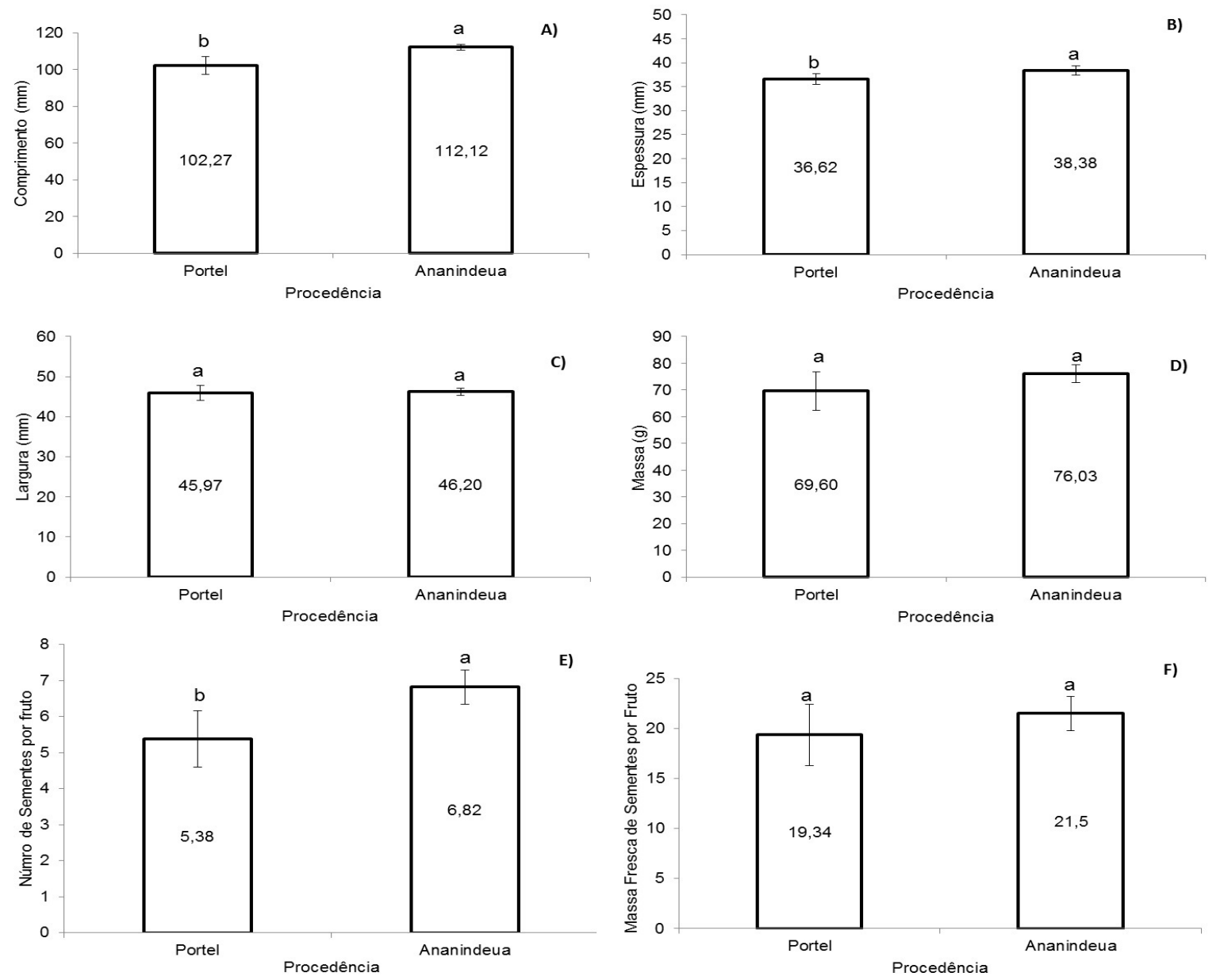

F)

FIGURA 1. Médias de Comprimento (A), Espessura (B), Largura (C), Massa Fresca (D), Número de Sementes por Frutos (E) e Massa fresca de Sementes por Frutos $(F)$ de frutos de duas procedências de jutaí-açú.

Informações sobre a biometria de sementes fornecem informações sobre a dispersão e estabelecimento de mudas nas florestas nativas (RICKLI-HORST et al., 2017). Por outro lado, essas informações podem auxiliar na determinação de procedências com variabilidade genética necessária para produção de mudas com fins de recuperação de áreas degradadas. Para a biometria das sementes observouse diferença significativa entre as procedências em relação ao comprimento (Figura 2A) e espessura (Figura 2B), sendo o município de Portel apresentou médias superiores (23,74 $\mathrm{mm}$ e $11,51 \mathrm{~mm}$, respectivamente) ao município de Ananindeua. Quanto à largura (Figura 2C), as duas procedências foram diferentes entre si, no entanto, o município de Ananindeua $(20,77 \mathrm{~mm})$ apresentou média superior as 
sementes de Portel. Não houve diferença significativa entre as duas procedências quanto à massa fresca das sementes (Figura 2D).
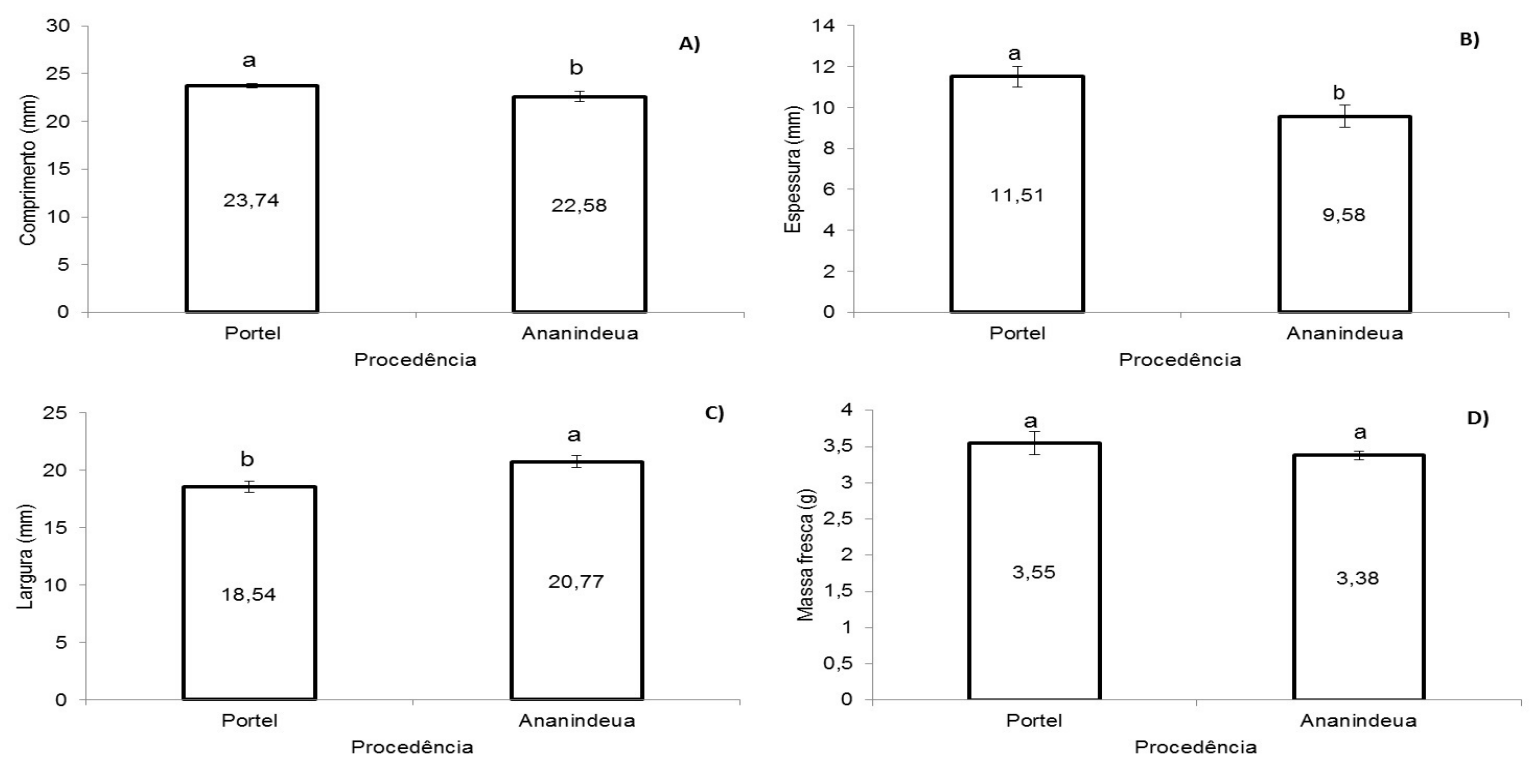

FIGURA 2. Médias de comprimento (A), espessura (B), largura (C), massa fresca (D) de sementes duas procedências de jutaí-açú.

Os resultados biométricos para frutos e sementes de Swietenia macrophylla King, coletados de 20 matrizes localizadas em diferentes municípios do sudeste do estado do Pará indicaram alta diversidade fenotípica, independentemente da localização da matriz (LEÃO et al., 2018). Com relação aos estudos de emergência de plântulas, houve diferença significativa entre as procedências para tempo médio de emergência (TME) e índice de velocidade de emergência (IVE), conforme observado na tabela 1.

TABELA 1. Médias de Porcentagem de Emergência (E), índice de Velocidade de emergência (IVE) e Tempo Médio de Emergência (TME) de Plântulas de jutaí-açú procedentes dos municípios de Portel e Ananindeua, PA.

\begin{tabular}{lccc}
\hline Procedências & $\mathrm{E}(\%)$ & IVE & TME \\
\hline Portel & $21 \mathrm{~b}^{*}$ & $0,23 \mathrm{~b}$ & $23,03 \mathrm{a}$ \\
Ananindeua & $73 \mathrm{a}$ & $1,27 \mathrm{a}$ & $14,86 \mathrm{~b}$ \\
\hline DMS & 9,58 & 0,18 & 3,34 \\
C.V $(\%)$ & 11,78 & 13,77 & 10,19 \\
\hline
\end{tabular}

${ }^{*}$ As médias com a mesma letra minúscula nas colunas não diferem estatisticamente pelo teste Tukey a $5 \%$.

A procedência de Ananindeua apresentou porcentagem de emergência $52 \%$ maior em relação à Portel (Tabela 1). Essa diferença observada entre as procedências pode estar relacionada com o grau de umidade das sementes ou fatores genéticos.

Estudos desenvolvidos por Pereira et al. (2015) com sementes de cinco procedências de Tecoma stans na região metropolitana de Belém, PA, também encontraram diferença significativa para as variáveis: porcentagem de germinação e índice de velocidade de germinação (IVG). Faria et al., (2009) afirmam que a procedência do lote pode influenciar nas características biométricas das sementes, ENCICLOPÉDIA BIOSFERA, Centro Científico Conhecer - Goiânia, v.15 n.27; p. 1292018 
no tempo médio de emergência e desenvolvimento das plântulas de Brosimum gaudichaudii.

\section{CONCLUSÃO}

O comprimento, espessura e número de sementes por fruto da procedência de Ananindeua foi superior aos valores médios de Portel, enquanto a largura, massa dos frutos e a massa fresca de sementes por fruto não diferiram entre as procedências. Todavia, em relação à morfometria das sementes, a procedência de Portel apresentou valores superiores para as variáveis de comprimento, espessura e massa fresca.

A procedência de Ananindeua apresenta qualidade fisiológica superior em relação à Portel, apresentando maiores médias para porcentagem e índice de velocidade de emergência e menor tempo médio de emergência.

Com base neste estudo recomenda-se o uso de testes de emergência de plântulas na seleção de árvores de Hymenaea courbaril com fins de colheita de sementes, para definir as árvores que produzam sementes com maior padrão de qualidade fisiológica.

\section{REFERÊNCIAS}

BRASIL. Ministério da Agricultura e da Reforma Agrária. Regras para análises de sementes. Brasília: SNDA/DNDV/CLAV, 399 p, 2009. Disponível em:

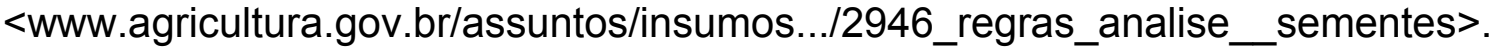

CIPRIANO J.; MARTINS, L.; DEUS, M. S. M.; PERON, A. N. O gênero Hymenaea e suas espécies mais importantes do ponto de vista econômico e medicinal para o brasil. Caderno de Pesquisa. Série Biologia, v. 26, n.2, p.41-51, 2014. Disponível em: <http://dx.doi.org/10. 17058/cp.v26i2.5248>. doi: 10.17058/cp.v26i2.5248.

DUARTE, R. I; SILV, F. A.L.S.; SCHULTZ, J. SILVA, J.Z.; REIS, M. S. Característica de desenvolvimento inicial em teste de progênie de uma população de Araucária na Flona de Três Barras- SC. Instituto Chico Mendes. Biodiversidade Brasileira, 2 (2), 114-123, 2012. Disponível em:

<http://www.icmbio.gov.br/revistaeletronica/index.php/BioBR/article/view/279>.

DUTRA, F.V.; CARDOSO, A. D.; SILVA, R. M. DA, LIMA, R.; MORAIS, O. M.; R. M. C. Morfobiometria de frutos e sementes de Schizolobium amazonicum Huber ex Ducke. Revista Agropecuária Técnica, Areia-PB, v. 38, n.2p.58-64, 2017. Disponível em: <http://dx.doi.org/ 10.25066/agrotec.v38i2.29456>. doi: 10.25066/agrotec.v38i2.29456.

FARIA, R. A. P. G.; SILVA, A.N.; ALBUQUERQUE, M.C.F.; COELHO, M.F.B. Características biométricas e emergência de plântulas de Brosimum gaudichaudii Trec. oriundas de diferentes procedências do cerrado mato-grossense. Revista Brasileira de Plantas Medicinais, Botucatu, v. 11, n. 4, p. 414-421, 2009. Disponível em: <http://dx.doi.org/10.1590/S1516-05722009000400009>. doi: 10.1590/S1516-05722009000400009.

FREITAS, A. D. D.; LEÃO, N. V. M.; DE POTIGUARA, R. C. V.; REIS, A. R. S. e SOUSA, D. V. Caracterização Morfológica do Fruto, Semente e Desenvolvimento Pós-Seminal de Aspidosperma spruceanum BENTH. EX MULL. ARG. (APOCYNACEAE). Enciclopédia Biosfera, Centro Científico Conhecer - Goiânia, v. 
10 , n.18, 2014.

Disponível

em:

$<$ http://www.conhecer.org.br/enciclop/2014a/AGRARIAS/caracterizacao

\%20morfologi ca\%20do.pdf>.

FAPESPA - Fundação Amazônica de Amparo a Estudos e Pesquisas - Estatísticas Municipais Paraenses. Diretoria de Estatística e de Tecnologia e Gestão da Informação. Belém, PA, 2016.2 Disponível em: <http://www.parasustentavel.pa.gov.br/downloads>.

GONÇALVES, L. G. V.; ANDRADE, F. R.; JUNIOR, M.; HUR, B.; SCHOSSLER, T. R.; LENZA, E.; MARIMON, B. S. Biometria de frutos e sementes de mangaba (Hancornia speciosa Gomes) em vegetação natural na região leste de Mato Grosso, Brasil. Revista de Ciências Agrárias, Lisboa, v. 36, n. 1, p. 31-40, jan. 2013. Disponível em: <http://www.scielo.mec.pt/scielo.php? script=sci_arttext\&pid=S0871018X2013000100006>.

GONZAGA, L. M.; SILVA, S. S. S.; CAMPOS, S. A.; FERREIRA, R. P.; CAMPOS, A. N. R.; CUNHA, A. C. M. C. M. Recipientes e substratos para a produção de mudas de jatobá (Hymenaea courbaril L.). Revista Brasileira de Agropecuária Sustentável, v. 6, n. 1, p. 64-73. 2016.

KOSZO, C. R. R. Germinação de sementes de Erythrina speciosa Andr. e Eugenia brasiliensis Lam. em meio ácido. Dissertação (Mestrado em Biodiversidade Vegetal e Meio Ambiente). Instituto de Botânica da Secretaria do Meio Ambiente, São Paulo. 2006. 84f.. Disponível em: <http://www.biodiversidade.pgibt.ibot.sp.gov.br/Web/teses/2006/Pdf/Cristina_Rita_R adics_Koszo_MS.pdf. >.

LEÃO, N. V. M.; FELIPE, S. H. S.; SILVA, C. E.; MORAES, A. C. S.; SHIMIZU, E. S. C.; GALLO, R.; FREITAS, A. D. D.; KATO, O. R. Morphometric diversity between fruits and seeds of mahogany trees (Swietenia macrophylla King.) from Parakanã Indigenous Land, Pará State, Brazil. Australian Journal of Crop Science, v. 12, p. 435-443, 2018.

Disponível

em:

<http://www.cropj.com/leao_12_3_2018_435_443.pdf>.

LIMA, W. A. A.; GREEN, M.; ZEVIANI, W. M.; LOPES, R.; RIOS, S. A. Teor de água e tempo de exposição ao tratamento térmico na germinação de sementes de caiaué. Revista de Ciências Agrárias/Amazonian Journal of Agricultural and Environmental Sciences, v. 60, n. 2, p. 192-198, 2017. Disponível em:<http://dx.doi.org/10.4322/rca.2625>. doi: 10.4322/rca.2625.

LORENZI, H.; Árvores brasileiras: Manual de identificação e cultivo de plantas arbóreas nativas do Brasil. Instituto Plantarum, Nova Odessa, 4. ed, v.1, 384 p. 2002.

LUCENA, F.R.; FERNANDES, H.E.; MIRANDA, R. V.; SOUZA, P.A. de.; PEREIRA, M.A. Influência do Sombreamento na germinação de sementes de Hymenaea courbaril L. Enciclopédia Biosfera, Centro Científico Conhecer - Goiânia, v.13 n.23; p. 2016. Disponível

em: 
<hhttps://dx.doi.org/10.18677/Enciclopedia_Biosfera_2016_060.

doi:

10.18677/Enciclopedia_Biosfera_2016_060.

MARTINS, S. V. Recuperação de áreas degradadas: ações em áreas de preservação permanente, voçorocas, taludes rodoviários e de mineração. 3 . ed. Viçosa: Aprenda fácil, 2013, v. 1. 264p.

PEREIRA, D. N.; MEDEIROS, A. P. R.; SANTOS, K. C. P.; TENÓRIO, R. S.; PIRES, H. C. G. Biometria e emergência de plântulas de ipê de jardim (Tecoma stans) oriundas de diferentes procedências da Região Metropolitana de Belém, Pará. Enciclopédia Biosfera, v. 12, p. 510-517, 2015. Disponível em: <http://dx.doi.org/10.18677/Enciclopedia_Biosfera_2015_104>. doi: 10.18677/Enciclopedia_Biosfera_2015_104.

RICKLI-HORST, H. C.; DUARTE, M. M.; CHIRINZANE, C. J.; NOGUEIRA, A. C. Carposeminal biometry and germination of Cabralea canjerana (Vell.) Mart. Floresta, v. 47, n. 4, p. 391-396, 2017. Disponível em:< http://dx.doi.org/10.5380/rf.v47i4.54128>. doi: 10.5380/rf.v47i4.54128.

SARMENTO, H.G.S.; SOUZA DAVID, A.M.S.; BARBOSA, M.G.; NOBRE, D.A.C.; AMARO, H.T.R. Determinação do teor de água em sementes de milho, feijão e pinhão-manso por métodos alternativos. Energia Na Agricultura, v.30, n.3, p.250256, 2015. Disponível em: <http://dx.doi.org/10.17224/EnergAgric.2015v30n3p250256. doi: 10.17224/EnergAgric.2015v30n3p250-256>.

SILVA, S.; LEÃO, N. V. M. Árvores da Amazônia. São Paulo: Empresa das Artes, 243 p. 2006.

SMIDERLE, O.J.; SOUSA, R.C.P. Dormência em semente de paricarana (Bowdichia virgilioides Kunth. - Fabaceae - Papilionidae). Pelotas. Revista Brasileira de Sementes. v. 25 n. 2. p. 2003. Disponível em: <http://www.scielo.br/pdf/ $\% 0 D / r b s / v 25 n 1 / 19633 . p d f>$.

ZUFFO, A.M.; ANDRADE, F.R.; ZUFFO JUNIOR, J. M. Caracterização biométrica de frutos e sementes de baru (Dipteryx alata Vog.) na região leste de Mato Grosso. Revista de Ciências Agrárias, Lisboa, v. 37, n. 4, p. 463-471, dez. 2014. Disponível em: <http://www.scielo.mec.pt/scielo.php? script=sci_arttext\&pid=S0871018X2014000400012>. 